\title{
Fermionic atoms in optical superlattices
}

\author{
B. Paredes, ${ }^{1}$ C. Tejedor, ${ }^{2}$ and J. I. Cirac ${ }^{1}$ \\ ${ }^{1}$ Max-Planck Institute for Quantum Optics, Garching, Germany \\ ${ }^{2}$ Dept. Física Teórica de la Materia Condensada, Universidad Autónoma de Madrid, Spain
}

(Dated: November 11, 2018)

\begin{abstract}
Fermionic atoms in an optical superlattice can realize a very peculiar Anderson lattice model in which impurities interact with each other through a discretized set of delocalized levels. We investigate the interplay between Kondo effect and magnetism under these finite-size features. We find that Kondo effect can dominate over magnetism depending on the parity of the number of particles per discretized set. We show how Kondo-induced resonances of measurable size can be observed through the atomic interference pattern.
\end{abstract}

PACS numbers: 03.75.Fi, 03.67.-a, 42.50.-p, 73.43.-f

Cold atoms in optical lattices are capturing a lot of attention from both experimental 1, 2] and theoretical sides [3, 4, 5, 6, 7, 8, 9]. This interest is highly motivated by the possibility of investigating the domain of strongly correlated phenomena, the interaction effects (typically small in free space) being enhanced due to the periodic confinement. As a unique feature of these atomic systems, the full control of the system's parameters allows to explore several fascinating directions. On the one hand, atoms in optical lattices can be used to provide illuminating and critical insight into models describing strongly correlated systems. For example, quantum phase transitions in both bosonic [3] and fermionic [5] Hubbard models, as well as in spin Hamiltonians [9], can be explored with unprecedent control. On the other hand, in what perhaps is even more challenging, exotic scenarios can be created in which strongly correlated phenomena may occur under novel conditions. A variety of possibilities already accessible experimentally (different lattice topologies created with superpositions of multiple laser beams [10], independent periodic potentials for different internal atomic states 2, 4], interactions controlled by Feshbach resonances [11], etc) can be combined, promising new ways to strongly entangle atomic ensembles.

In this letter we study the physics of fermionic atoms in an optical superlattice 10. We will show that the system realizes an Anderson Lattice Hamiltonian (ALH) [12]. The ALH has been extensively studied in the context of strongly correlated electrons [13, 14], and is known to capture the physics of a variety of strongly correlated phenomena, from Kondo effect 13 to RKKY magnetism 13, 14]. Typical condensed matter systems described by Anderson models are metallic or intermetallic compounds with a low concentration of magnetic impurities. The usual scenario is then that of impurities located far from each other, each of them coupled to a continuum of delocalized electrons. In an interesting volte-face, atoms in a superlattice naturally realize a quite different situation, allowing us to investigate a very peculiar regime. For realistic experimental situations supersites (that will play the role of impurities) will be separated typically by a small number of lattice sites. Therefore, (if, for instance, tunneling is only allowed along one direction), the system realizes an array of impurities connected through small "islands" with a discretized set of levels. The situation resembles that of an array of the Kondo boxes theoretically studied in 15], where impurities can now interact with each other through the intermediate "conducting islands". We will show that, in such a situation, both Kondo effect and the competition between Kondo effect and magnetism are strongly affected by finite-size effects, with a remarkable enhancement of the Kondo temperature. We explain how to induce and observe the strongly correlated effects we predict by combining several different techniques.

We consider a gas of fermionic atoms embedded in a superlattice of period $L$ with potential depth $V_{0}$ for "normal" sites and $V_{0}^{\prime}$ for "supersites" (see Fig.1). We assume that two kinds of atoms are present (generalized spin $\sigma=\uparrow, \downarrow)$. For sufficiently low temperatures atoms will

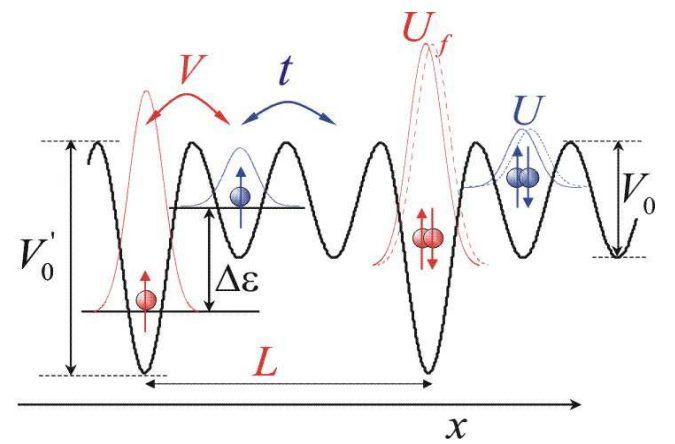

FIG. 1: AHM for atoms in a superlattice (see text).

be confined to the lowest Bloch band of the superlattice and the system can be described by an ALH of the form

$$
\begin{aligned}
H_{A L H}= & -t \sum_{<\ell, \ell^{\prime}>\sigma} c_{\ell \sigma}^{\dagger} c_{\ell^{\prime} \sigma}+U \sum_{\ell} n_{\ell \uparrow} n_{\ell \downarrow}-\Delta \epsilon \sum_{s \sigma} n_{s \sigma}^{f} \\
& +U_{f} \sum_{s} n_{s \uparrow}^{f} n_{s \downarrow}^{f}+V \sum_{<\ell, s>\sigma}\left(f_{s \sigma}^{\dagger} c_{\ell \sigma}+\text { h.c }\right),(1)
\end{aligned}
$$

where $c_{\ell \sigma}, f_{s \sigma}$ are fermionic operators that annihilate an atom with spin state $\sigma$ on normal site $\ell$ and supersite $s$, respectively, and $n_{\ell \sigma}=c_{\ell \sigma}^{\dagger} c_{\ell \sigma}, n_{s \sigma}^{f}=f_{s \sigma}^{\dagger} f_{s \sigma}$ [19]. 
The Hamiltonian (11) has been extensively studied in the context of strongly correlated electrons. It is well known that in the regime in which $U_{f}, \Delta \epsilon \gg t, V \gg$ $U$, the so called Kondo regime [16], strongly correlated effects appear. Within this regime the low-energy physics of Hamiltonian (11) can be described by an effective model in which the $f$-atoms degrees of freedom are represented by localized spins, the well known Kondo lattice model (KLM) 13, 16],

$$
H_{K L M}=-t \sum_{<\ell, \ell^{\prime}>\sigma} c_{\ell \sigma}^{\dagger} c_{\ell^{\prime} \sigma}+J \sum_{s} \mathbf{S}_{s}^{f} \cdot \mathbf{S}_{s}^{c},
$$

where $\mathbf{S}_{s}^{f}=\frac{1}{2} \sum_{\sigma, \sigma^{\prime}} \tau_{\sigma, \sigma^{\prime}} f_{s \sigma}^{\dagger} f_{s \sigma^{\prime}}$ are localized spins and $\mathbf{S}_{s}^{c}=\frac{1}{2} \sum_{\sigma, \sigma^{\prime}} \tau_{\sigma, \sigma^{\prime}} d_{s \sigma}^{\dagger} d_{s \sigma^{\prime}}$ with $d_{s \sigma}=\sum_{\langle s, \ell\rangle} c_{\ell \sigma}, \tau$ being the vector of Pauli matrices. The exchange interaction $J=2 V^{2} / \Delta \epsilon$ is antiferromagnetic, and though typically very small $(J \ll t)$ in condensed matter systems, is the source of interesting many-body effects. The KLM has been studied typically in two different situations [16]. 1) A single localized spin weakly coupled to a continuum, which is the usual Kondo problem [13, 14. Here it is well known that for temperatures bellow a critical temperature $T_{K}$, the Kondo temperature, a many-body singlet is formed composed of the localized spin and the local spin polarization of conduction electrons. As a result of singlet formation a resonance appears, the Kondo resonance, which is responsible of many interesting effects. As a remarkable feature, Kondo effect is non-perturbative in $J$, with a characteristic exponential behavior of the Kondo temperature $T_{K} \sim 2 t e^{-1 / 2 J \rho\left(\epsilon_{F}\right)}$, where $\rho\left(\epsilon_{F}\right)$ is the density of states at the Fermi level. The case of a single impurity coupled to a small metallic grain has been also theoretically studied in 15. Here it is found that the Kondo resonance can be strongly affected by the finite size of the grain. 2) A Kondo lattice with typically one impurity per conduction electron. In this case interaction between localized spins (the RKKY interaction, with a characteristic long range oscillatory behavior) can take place, mediated by the continuum of conduction electrons. Competition between local Kondo singlet formation and RKKY magnetism has been investigated as the ratio $t / J$ is varied [16]. Since magnetism is a perturbative effect in $J$, it is predicted to dominate over Kondo effect in the weak coupling regime $(J \ll t)[16]$.

The atomic system that we have under consideration can realize a situation different from the cases described above. Let us assume that tunneling is only allowed along one direction (potential barriers have been made very high in the other directions) and that the parameters in (11) fulfill the conditions to be in the Kondo regime. The system realizes then a 1D Kondo lattice in which impurities interact with each other through a discretized set of levels. A new characteristic energy scale appears, namely the separation between levels of an island, $\Delta$. For $\nu=1 / 2(\nu=M / N, N$ being the number of particles and $M$ the total number of sites), the separation of the Fermi level and the next excitation is $\Delta=2 t \sin (\pi / L)$, which is finite in our case. In addition, the ratio $t / J$ (as we show later) can be varied, so that discussion of both the strong and weak coupling limits is experimentally relevant in this case.

Strong coupling regime: $J \gg \Delta$. Kondo screening dominates the physics of the problem. Since tunneling is very small, impurities are basically disconnected from each other and singlet formation occurs independently for each of them. In this limit we can use a generalization of the variational wave function of Varma and Yafet [17] for the ground state:

$$
|\Psi\rangle=\prod_{s}\left(\beta+\sum_{k} \beta_{k}\left(f_{s \uparrow}^{\dagger} A_{s k \uparrow}+f_{s \downarrow}^{\dagger} A_{s k \downarrow}\right)|F S\rangle_{s}\right),
$$

where $A_{s k \sigma}=\sqrt{\frac{2}{L}} \sum_{\langle s, \ell \sigma\rangle} \sin (k \ell) c_{\ell \sigma}, k=\pi n / L, n=$ $1, \ldots, L-1$, and $|F S\rangle_{s}=\prod_{k, \sigma}^{k_{F}} A_{s k \sigma}^{\dagger}$ with $k_{F}=$ $\pi N / 2 L$. The variational coefficients $\beta$ and $\beta_{k}$ satisfy $|\beta|^{2}+2 \sum_{k}\left|\beta_{k}\right|^{2}=1$. For each $f$-site, (3) describes singlet formation with a delocalized state of momentum $k$ with an amplitude given by $\beta_{k}$. Minimization of $E=\langle\Psi|H| \Psi\rangle /\langle\Psi \mid \Psi\rangle$ with respect to the $\beta$ 's yields: $\beta_{k} / \beta=-\frac{2}{\sqrt{L}} V \sin k /\left(T_{K}+\Delta_{k}\right)$, and a Kondo temperature given by the implicit equation

$$
T_{K}=\Delta \epsilon-\epsilon\left(k_{F}\right)+\frac{16 V^{2}}{L} \sum_{k}^{k_{F}} \frac{\sin ^{2} k}{T_{K}+\Delta_{k}},
$$

where $\Delta_{k}=\epsilon\left(k_{F}\right)-\epsilon(k), \epsilon(k)=-2 t \cos k$. Under realistic conditions we will have $L<10$ and the sum in (4) will be a discrete sum with a few $(\sim L / 2$ for $\nu=1 / 2)$ terms. As a result, $T_{K}$ does not go to zero with $t / J$ (Figure 2), but remains $\sim J$ due to the finite size of the conducting island. When $\Delta$ becomes of the order of $J$ the size of the singlets becomes comparable to the separation $L$ between supersites, so that the screening cloud of one impurity starts affecting the next supersite. An interplay between Kondo effect and magnetism takes place.

Weak coupling regime $\Delta \gg J$. A very different situation corresponds to the regime in which the spacing between energy levels in the conducting islands $(\sim$ $2 t \sin (\pi / L)$ ) becomes much larger that the Kondo temperature $(\sim J)$. Within this limit atomic orbital degrees of freedom are completely frozen, with excitations above the Fermi level in each of the islands taking part of the problem only as virtual states. Performing adiabatic elimination of these excitations in Hamiltonian (2), we obtain an effective Hamiltonian for the spin degrees of freedom. As an interesting feature the resulting Hamiltonian depends on the parity of the number of particles per conducting island, $N_{c}$. This even-odd effect is a clear manifestation of the finite size of the conducting islands.

$N_{c}=$ even . The Fermi level of each island is occupied by two atoms. In this case the only spin degrees of freedom correspond to atoms localized in supersites. An effective spin-spin interaction between neighboring supersites appears, mediated by the Fermi sea in between them. To second order perturbation theory in $J$, 


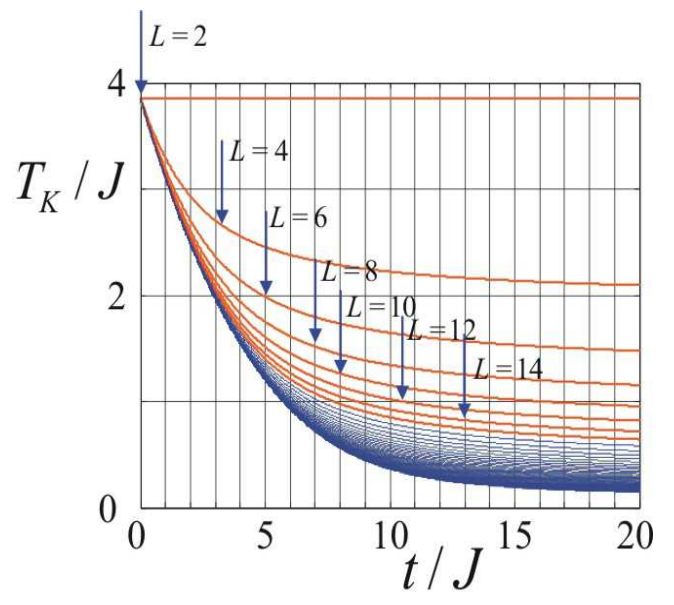

FIG. 2: Kondo temperature $T_{K}$ as a function of $J / t$. Different curves correspond to increasing values of $L(N=L / 2)$. For realistic cases (in orange), the arrows mark points at $T_{K}=$ $2 \Delta$, to the right of which expression (4) is not valid.

$$
H_{S S}=J_{e f f}^{M} \sum_{\left\langle s, s^{\prime}\right\rangle} \mathbf{S}_{s}^{f} \cdot \mathbf{S}_{s^{\prime}}^{f},
$$

where $J_{\text {eff }}^{M}=\frac{J^{2}}{t L} \sin \left(k_{F}\right) \sin \left(k_{F}(L-1)\right) \sin \left(k_{F}+\right.$ $\Delta k) \sin \left(\left(k_{F}+\Delta k\right)(L-1)\right)$, and $\Delta k=\pi / L$. We see that Kondo effect disappears and magnetism is induced for localized atoms, the ground state being antiferromagnetic or ferromagnetic depending on both $L$ and $k_{F}$. We note that due to the characteristic topology imprinted by the superlattice, Heisenberg (and not RKKY) magnetism is induced.

$N_{c}=$ odd. The Fermi level of each island is occupied by one atom, whose spin comes into play. The effective Hamiltonian is in this case $H=J_{e f f}^{K} \sum_{s} \mathbf{S}_{s}^{f} \cdot \mathbf{S}_{s}^{k_{F}}$, where $\mathbf{S}_{s}^{k_{F}}=\frac{1}{2} \sum_{\sigma, \sigma^{\prime}} \tau_{\sigma, \sigma^{\prime}} A_{s k_{F} \sigma}^{\dagger} A_{s k_{F} \sigma^{\prime}}$, and $J_{\text {eff }}^{K}=4 J / L$. Kondo effect remains in this case and magnetism is not induced. The ground state consists in this case of singlets formed by each localized atom and the atoms at the Fermi level in neighboring islands. The Kondo temperature is $T=2 J_{\text {eff }}^{K}$.

Numerical results. To illustrate the predictions above we have numerically diagonalized Hamiltonian (1) for a small 1D superlattice. In Fig. 3 and Fig. 4 we plot the spin-spin correlation functions $\left\langle\mathbf{S}_{f} \cdot \mathbf{S}_{\ell}\right\rangle$ (spatial correlation of a fixed $f$-spin with the rest of sites in the chain, $\ell$ ), and $\left\langle\mathbf{S}_{f} \cdot \mathbf{S}_{k}\right\rangle$ (correlation of a fixed $f$-spin with a delocalized spin with momentum $k$ ) for the exact ground state. We consider different cases. a) $L=4, N_{c}=4$ (Fig. 3). Fig. 3a) shows a clear smooth transition from local Kondo singlet formation to magnetism of localized spins. For small values of $t / J$ each localized $f$-spin is antiferromagnetically correlated with its next neighboring sites (forming a singlet with them). As $t / J$ increases correlations of each impurity with its neighboring islands disappear, at the same time that correlations between next supersites are induced. The transition (arrow in inset of Fig. 3a)) takes place around $T_{K} \sim 2 \Delta(t / J \sim 3.25)$

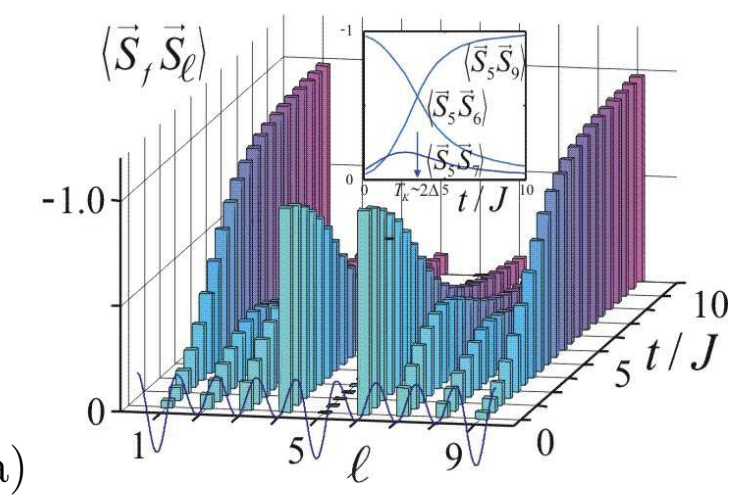

b)

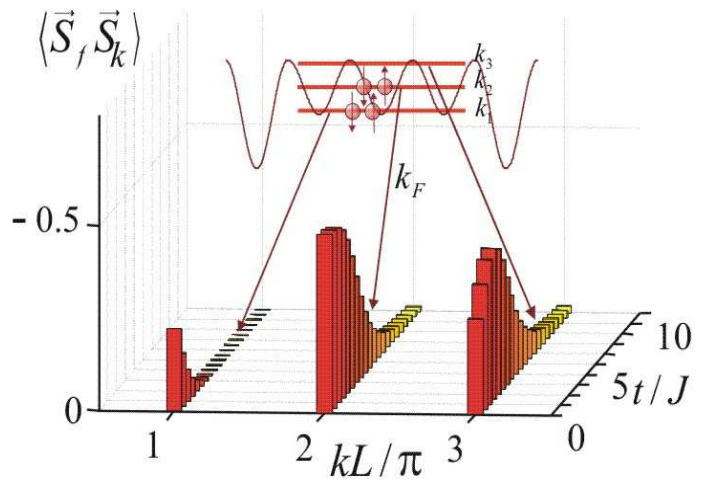

FIG. 3: Spin-spin correlation functions of a fixed f-site $(s=$ 5 ) with the rest of sites (a) and the k-momentum states of a neighboring island (b), as a function of $t / J$. Parameters: $M=9, N=11\left(N_{c}=4\right), U=0, U_{f}=10 \Delta \epsilon, \Delta \epsilon=10 \mathrm{~J}$. In the inset of a) correlations of supersite 5 with sites 6,7 , and supersite 9 are plotted.

as predicted. As stated by Hamiltonian (15) impurities are antiferromagnetically coupled $\left(J_{\text {eff }}^{M}=-J^{2} / 16 t\right)$. b) $L=4, N_{c}=3$ (Fig. 4). Kondo effect appears in this case as $t / J$ increases. In real space Fig. 4a) shows how singlet-type correlations become more and more extended along the conducting islands next to each impurity, whereas neighboring impurities remain uncorrelated. Delocalization of the singlet becomes more evident in momentum space (Fig. 4b), where a resonance, the Kondo resonance, appears at the Fermi level. The Kondo temperature is always of the order of $J$ (see inset of Fig. 4b), reaching the limiting value $T \sim 2 J_{\text {eff }}^{K}=2 J$ for $J \ll \Delta$, as predicted above.

We discuss now the experimental realization of the regimes we have studied above. First of all we need $U_{f} \gg U$. Since $U_{f} / U \sim V_{0}^{\prime} / V_{0}$ the potential depth of supersites must be very large. As a consequence the energy offset $\Delta \epsilon \sim V_{0}^{\prime}-V_{0}$ will be also large whereas the coupling $V$ will be very small. For a typical value of $V_{0}^{\prime} / V_{0} \sim 10$ we obtain $J<10^{-4} t$, which yields extremely small Kondo temperatures, completely unrealistic with current technology. In order to overcome this problem we propose the following scheme. Let us consider an ensemble of ${ }^{6} \mathrm{Li}$ atoms, which have six hyperfine states 


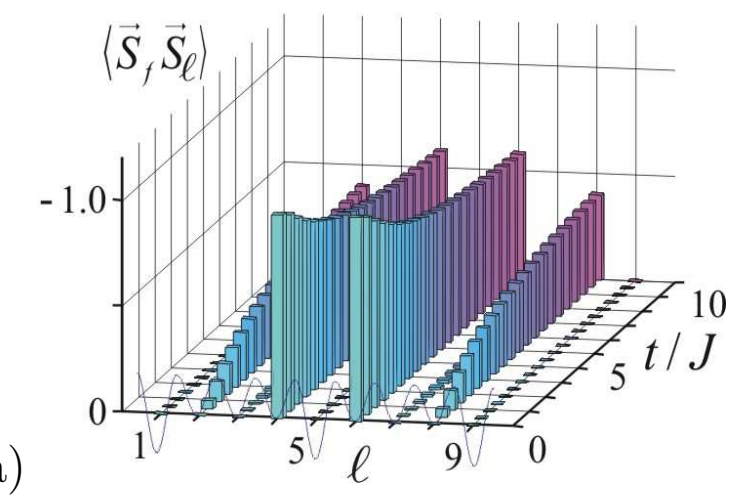

a)

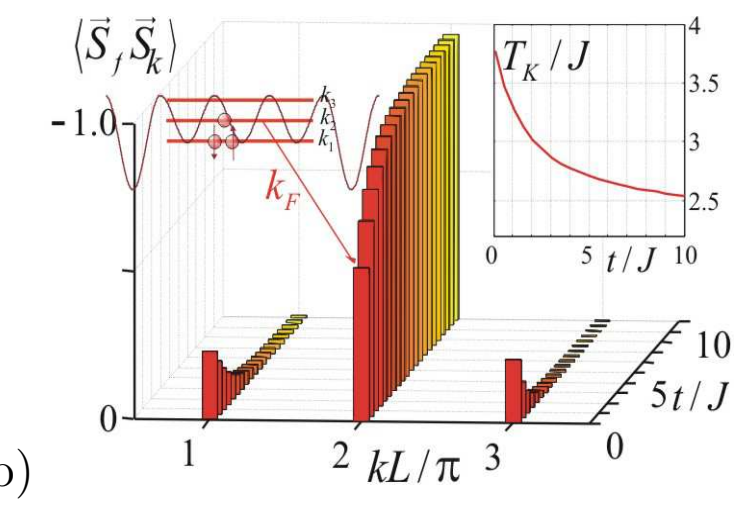

FIG. 4: Same as in Fig. 3 with $N=9\left(N_{c}=3\right)$. The Kondo temperature is plotted in the inset of $b)$.

$\left|F, M_{F}\right\rangle$, with a total spin $F=1 / 2$, or $3 / 2[18$. We consider a situation in which four of these internal states, $a \uparrow, a \downarrow, b \uparrow, b \downarrow$ are trapped (as shown in Fig 5.). We will also assume that interactions are engineered in such a way that interactions of type $a-a$ and $a-b$ are negligible, whereas $b-b$ are positive and large. The goal is to use a-atoms (non-interacting) as "conducting" c-atoms, and b-atoms (strongly interacting) as localized f-atoms. One possibility is the following. Let us assume we load ${ }^{6} \mathrm{Li}$ atoms in an optical lattice, with $N_{\uparrow}=N_{\downarrow}, \nu_{b}=1$, and $\nu_{a}<1$ (variable). Atoms of type $a$ will delocalize along the lattice forming a Fermi sea, whereas atoms of type $b$ will be localized forming a Mott phase. If the superlattice is now adiabatically turned on supersites will be filled up, each supersite containing two $a$-atoms with opposite spins and only one $b$-atom. The idea is to couple $a$-atoms in normal sites with $b$-atoms in supersites by using an off-resonant laser. By tuning the intensity $\Omega$ and frequency $D$ of the laser, the parameters $\Delta \epsilon$ and $V$ can be tuned to the desired values. The only restriction here is that the laser must not excite other processes, as for example, transitions between a-atoms and b-atoms in normal sites. This sets the condition $(\Omega / \delta)^{2} \ll 1$ (see Fig. 5). Taking this into account it is possible to make $0.5<t / J<20$, with values of $J \sim 0.1 E_{R}$. This yields Kondo temperatures $T_{K} \sim \mu K$, which are of the order of the ones required to observe superfluidity for fermionic atoms in optical lattices [5]. Finally, we discuss realiza- tion of the superlattice. Each of the Fourier components of the superlattice $\left(e^{i k_{n} x}\right.$, with $k_{n}=k n / L, n$ integer) can be realized experimentally by two counter-propagating laser beams with wave vector $k$ forming an appropriate angle $\theta_{n}$. Therefore the number of lasers required for a good realization of the superlattice increases with $L$ (for $V_{0}^{\prime} / V_{0} \sim 4$ a set of $\sim 2 L$ lasers is needed) But, as we have shown, systems with $L=3,4$ already display the strongly correlated phenomena we have predicted.

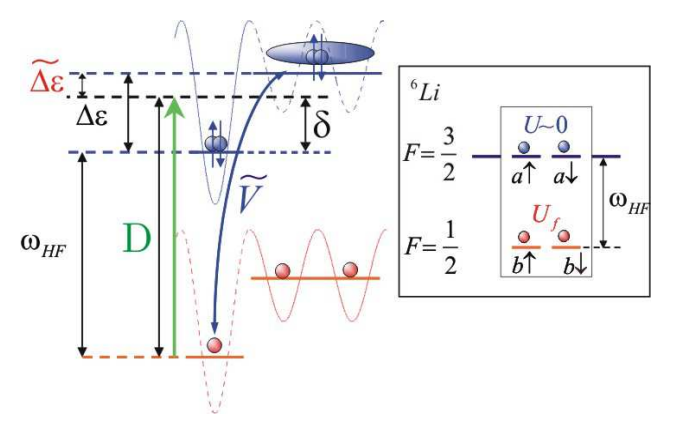

FIG. 5: Experimental scheme (see text). The renormalized parameters are $\widetilde{\Delta \epsilon}=\omega_{H F}+\Delta \epsilon-D$ (with $\omega_{H F}$ the hyperfine frequency), and $\widetilde{V}=\Omega V$.

Experimental observation. One of the most clear manifestations of Kondo effect is the appearance of Kondoinduced resonances in several physical magnitudes, as for instance spin-spin correlation functions. These correlations may be however hard to measure in actual experiments, since they involve two particle correlations. Instead, we propose to measure the one-particle correlation function $\left\langle A_{k \sigma}^{\dagger} f_{\sigma}\right\rangle$. From (3) we get $\left\langle A_{k \sigma}^{\dagger} f_{\sigma}\right\rangle \sim \beta \beta_{k}$, so that the localization of the singlet in the vicinity of the Fermi level (Kondo resonance) will show up in this quantity. This correlation can be obtained in the following way. Both $\left\langle c_{\sigma \ell}^{\dagger} c_{\sigma \ell^{\prime}}\right\rangle$ and $\left\langle f_{\sigma s}^{\dagger} f_{\sigma s^{\prime}}\right\rangle$ can be detected in the interference pattern measured after free expansion of $a$ and $b$-atoms, respectively. As well, by applying a $\pi / 4$ laser pulse between the internal states $a$ and $b$ right before measurement, $\left\langle\left(f^{\dagger}+c^{\dagger}\right)(f+c)\right\rangle$ can be obtained. Combining these three we obtain the desired correlation. Finally, magnetism between impurities can be detected by using spin dependent Bragg scattering, which will show up the antiferromagnetic or ferromagnetic order of the localized spins.

In conclusion we have shown that fermionic atoms in optical superlattices exhibit strongly correlated phases, from Kondo singlet formation to magnetism of localized spins. Characteristic features of this system are the finite size of the conducting islands coupled to supersites, which strongly influence the competition between Kondo effect and magnetism. These entangled phases could be used for atomic spintronics and spin-filtering.

Discussions with G. Gómez-Santos and J. von Delft are gratefully acknowledged. 
[1] M. Greiner et al., Nature (London) 415, 39 (2002); M. Greiner et al., Nature (London) 419, 51 (2002).

[2] O. Mandel et al., cond-mat/0301169

[3] D. Jaksch et al., Phys. Rev. Lett. 81, 3108 (1998).

[4] D. Jaksch et al., Phys. Rev. A 65, 033625 (2002).

[5] W. Hofstetter et al., Phys. Rev. Lett. 89, 220407 (2002).

[6] B. Paredes et al., Phys. Rev. Lett. 90, 150402 (2003).

[7] B. Damski et al., Phys. Rev. Lett. 90, 110401 (2003).

[8] D. Jaksch et al., Phys. Rev. Lett. 89, 040402 (2002).

[9] E. Jané et al., Phys. Rev. A 65, 050302 (2002).

[10] L. Guidoni et al., Phys. Rev. A 57, R1501 (1998); A. Görlitz et al. Phys. Rev. A 64, 011401 (2001).

[11] S. Inouye et al., Nature (London) 392, 151 (1998); E. Timmermans et al., Phys. Rev. Lett. 87, 240403 (2001).

[12] P. W. Anderson, Phys. Rev. 124, 41 (1961).

[13] See, for example, P. Fulde, in Electron Correlations in
Molecules and Solids (Springer-Verlag, Berlin, 1995).

[14] A. C. Hewson, in The Kondo problem to Heavy fermions (Cambridge University Press, Cambridge, UK, 1993).

[15] W. B. Thimm et al., Phys. Rev. Lett. 82, 2143 (1999).

[16] H. Tsunetsugu et al., Rev. Mod. Phys. 69, 809 (1997).

[17] C. M. Varma, Y. Yafet, Phys. Rev. B 13, 2950 (1976); K. Yoshida, Phys. Rev. B, 147, 223 (1966).

[18] M. Houbiers et al., Phys. Rev. A 56, 4864 (1997).

[19] Considering Gaussian wave packets we have: $t=3 \eta^{2} e^{-(\pi \eta / 2)^{2}} E_{R}, \quad U=a_{s} k \sqrt{8 / \pi} \eta^{3}, \quad U_{f}=$ $U\left(V_{0}^{\prime} / V_{0}\right)^{3 / 4}, \quad \Delta \epsilon=\left[\eta^{4}\left(\gamma^{4}-1\right)-3 \eta^{2}\left(\gamma^{2}-1\right)\right] E_{R}$, $V=\sqrt{2} \beta e^{-(\pi \eta \beta)^{2} / 4} t$, with $E_{R}=\hbar^{2} k_{\text {laser }}^{2} / 2 m$, $\gamma=\left(V_{0}^{\prime} / V_{0}\right)^{1 / 4}, \beta=\gamma /\left(1+\gamma^{2}\right)^{1 / 2}$. 